\title{
A Comparitive Analysis of Centralised and Decentralised Nurse Station and Patient's Satisfaction
}

\author{
Shaikh Javaria Manzoor \\ M.Arch, PhD student Graduate School of Architecture Hanyang University, ERICA Ansan-si 426-791 Sa 3-dong, Korea \\ *Corresponding Author: javeria@hanyang.ac.kr
}

Copyright (C) 2013 Horizon Research Publishing All rights reserved.

\begin{abstract}
This paper investigates on space syntax depth map software case studies of ICU design for four plan typologies, for investigation of the nurse station location, the layout design for the typology is been analyses on the space syntax program. There is a vacuum in the field of the Nurse Station location and ICU spatial composition. Since Evidence-Based Design (EBD) is the deliberately attempting to base ICU layout building decisions on the best available evidence with the goal of achieving the best possible outcomes 1 . The aim of this paper is that the hospitals in Karachi should have increased of number of single room accommodation. Moreover the typical ICUs is suggested to be to larger bed spaces $=20$ meter square. ICUs may have $10-20 \%$ single rooms $\mathrm{A}$ healing environment is the result of a design that has demonstrated measurable improvements in the physical and/or psychological states of patients and/or staff, physicians, and visitors. To identify the mechanisms by which sun light impacts child human health and performance and review the literature linking daylight with health outcomes in Pakistan healthcare settings. A window may provide variable degree of comfort for hospital buildings as that of high energy consumption hospitals. Window is a physical aspect in hospital design which creates a healing environment. This paper validates the role of daylight on patients satisfaction.
\end{abstract}

Keywords Day light, sky light, spectrum luminance, ultra violet $U V$ radiation calculation

\section{Introduction}

A three month quasi experiment was conducted, on site by author after survey. The patients at day care center were invited to participate in a questionnaire regarding the aesthetics of the day care space in relationship to the healing, and well- being amongst the patients. The study was approved by the Civil Hospital Karachi and hospital's Public Relations Committee for Medical and Healthcare
Research Ethics and the privacy ombudsman for the research at the Civil hospital Data Service for facilitating the data collection. More than 100 answers have been analyzed statistically by MS Excel program.

"A window design, can provide same or even more (i.e. variable degree) level of comfort for hospital buildings as that of high energy consumption hospitals"

\section{Materials and Methods}

Methods: Determinants of day light design has been identified in the pediatric burns ward which is a case study for this research. For verification a field survey was conducted along with a designed questionnaires which was filled by patients, nurses and visiting families. This study is based on the Ulrich proposal that: a view of natural elements serves to evokes positive emotions and manages stresses.

Results: Key findings are the quantitative observation and opinion compared with numerical data of luminance of daylight in the ward environment with regard to vision comfort. For social, physical and symbolic healing luminous environment is an integral aspect for hospital healing environment. Implications: Previous scientific studies by Ulrich have proved that surgery patients having views to nature in ward spent less time in hospital.

Figure 1 shows the position of the nurse station in red colors building types; yellow represents the corridor or circulation area. Blue represents ward typology. Green here refers to nurse station.

Hence it could be observed in figure 2 the centralized location of nurse station which is preferred and is used in pediatric department at civil hospital. Whereas 3 represent the lateral bay system which is observed in the burns department. exposed to $46 \%$ higher-intensity sunlight on average. This study found that patients exposed to an increased intensity of sunlight experienced less perceived stress, marginally less pain, took $22 \%$ less analgesic medication per hour, and had $21 \%$ less pain medication costs [14]. 


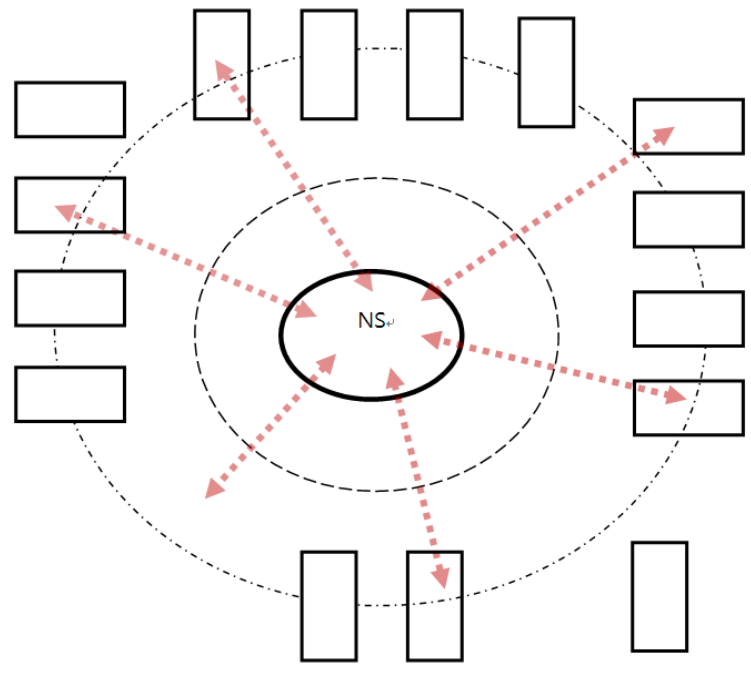

Figure 1. typical centralized nurse station planning

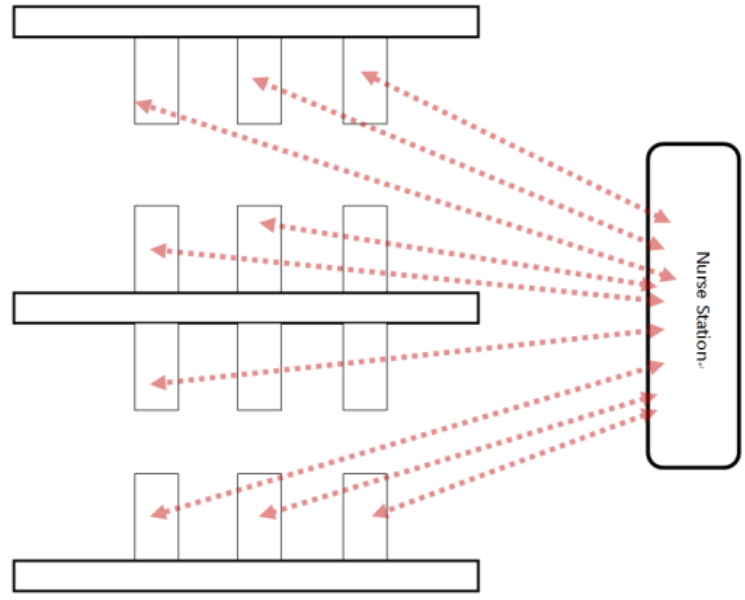

Figure 2. lateral view of the patients

Figure 1 and 2 shows the position of the pediatric nurse station for both the building types. Hence it could be observed in figure 2 the centralized location of nurse station which is preferred and is used in pediatric department at civil hospital. Whereas Figure 3 represent the lateral bay system which is observed in the burns department.

\section{Literature Review}

"Evidence-based medicine is the conscientious, explicit and judicious use of current best evidence in making decisions about the care of individual patients." [3]

As far as functionality and technology for the EBD healing environment is concerned, Acuity-adaptable care model challenge [1] main purpose of acuity-adaptable care is to reduce the requirement to transfer patients during their hospital stay. The patient stays in the same room or unit retains the continuity of the care team, which will result in reduce medical errors, improve operational efficiencies, and increase patient satisfaction.

Decentralized nurse station design, since nurses, by nature, are social beings and they like being with other people, could also experience some sense of isolation working in the new decentralized model. Whereas on the other hand personal communication devices team-based centralized structures, and spaces for team meetings and conference might helped to reduce this problem.[1]

Technology and IT has played a vital role for the ICU design, ease to communicate, and the facility to connect to internet through several devices has made healthcare facility more efficient, the intelligent hospital system used these facilities to have instant cure to the patient, and the ease to be connected to the Central Hub for the information of the patient and the entire medical history. Rapid Response Team is available for the instant accurate specialized treatment.

The Design multi-disciplinary Team

ICU design is a multidisciplinary team of, comprising of the ICU medical director, ICU nurse manager, the chief architect, hospital administration, and the operating engineering staff [2]. The architect should be specialized in hospital functional planning; the engineers must be specialized in the design of mechanical and electrical systems for hospitals, for critical care units. The design team should be expanded by adding members of other hospital administration of critical care unit, to insure that the design meets its intended function. Moreover environmental engineers, interior designers, staff nurses, physicians, and patients and families could be asked for questionnaire on how to provide a user-friendly environment. The ability to provide specific levels of care must be determined by analyzing physician resources, staff resources (nursing, respiratory therapy, etc), and the availability of support services (Laboratory, radiology, pharmacy, etc.) [2].

"The patient room is a key element of a hospital. We instigated a continuing education exercise to stimulate discussion about what will be important to the hospital room during the next several decades," says John Waugh, AIA, Ellerbe Becket principal architect [4].

There is a need to broaden the collaborative sensibilities in holistic value-driven design. To aim for ICU design should be to explore the bridge between functional efficiency and a humane supportive environment. To solve qualitative design challenges without sacrificing functional performance [5].

Single- vs. Multi-Bed Rooms choice, research shows that single rooms are superior to multi-bed rooms in terms of patient safety. They also enhance more privacy. Rooms providing full enclosure have shown to increase sleep quality.

Critical care facility design has an impact on the performance of organization, clinical results, and care delivery cost. Hence best current evidence is advised to be involved in design and construction projects [6].

\section{Case study}


Literature review suggests that there is constant need to rigorous revision of ICU design, since the IT and intelligent building design phenomena is revising constantly. Hence architects have to keep revising the space program of spatial composition of ICU in Korea. Moreover there is a need for a multi-disciplinary approach to be incorporated where ergonomic specialist for the ICU, patient interaction as well as the staff and machine interface. The usability and use experience both from patient as well as nurse point of view is been analyzed here.

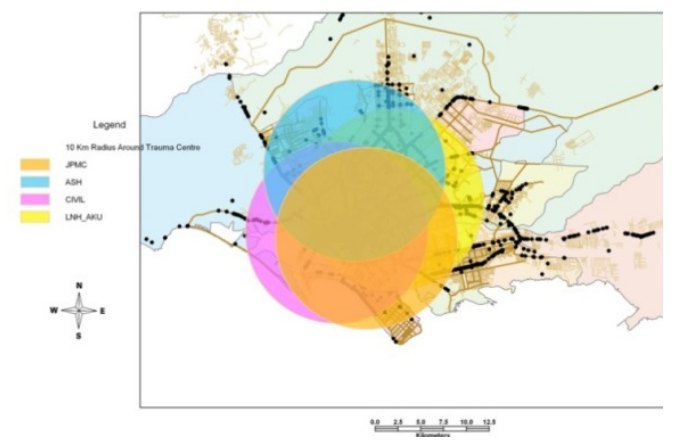

Figure 4. shows the site plan of Karachi with the major hospitals.

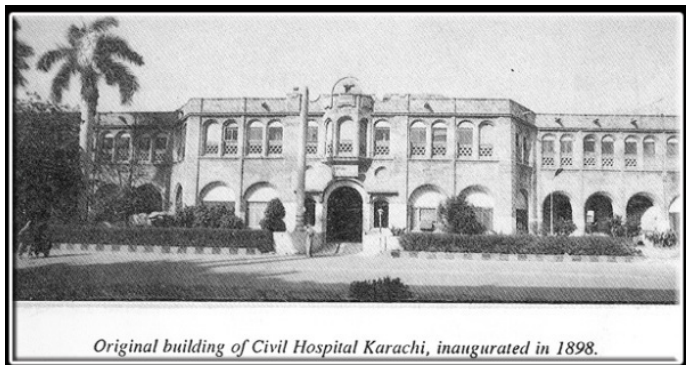

Figure5. Civil Hospital Karachi: institutional building type

\section{1 Historical background}

The Civil Hospital Karachi was founded in 1898, Initially established in 1898 with 250 beds to provide basic health facilities,

Comparative analyses, of the outer bay, of two similar building, at Civil hospital Karachi, one after renovation, and other without renovation.
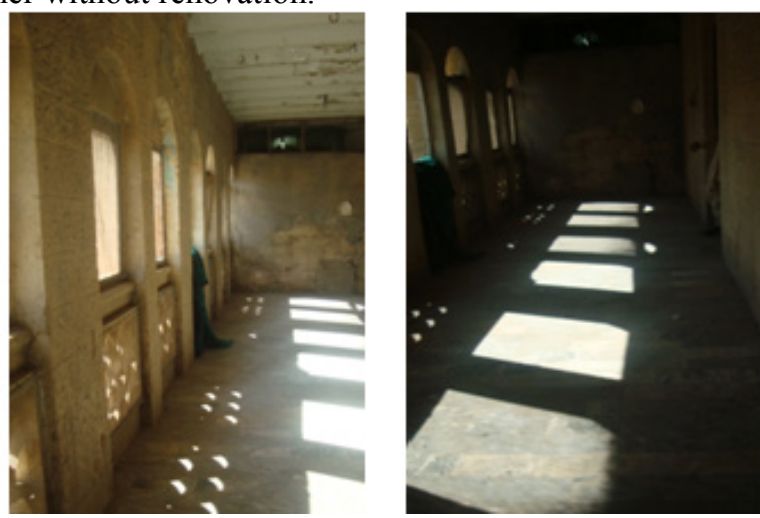

Figure 6. The balcony of the building brining in the UV lights for the patients

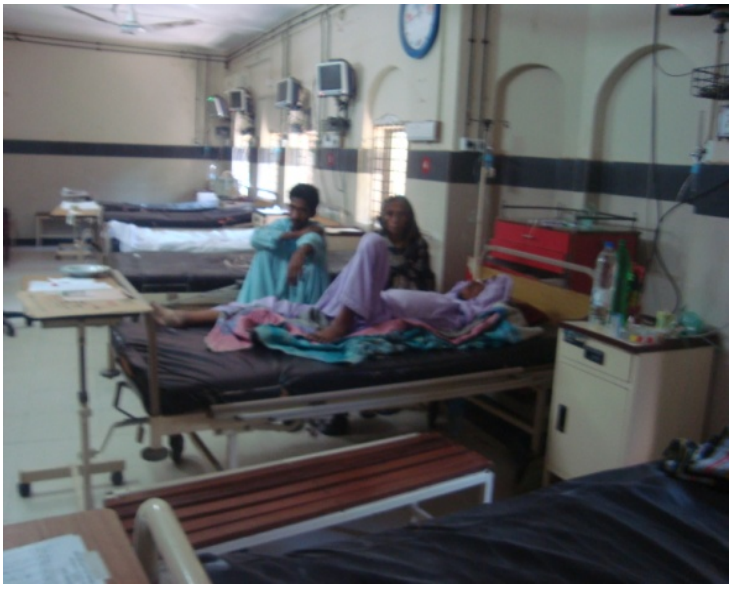

Figure 7. the nightingale type ward at civil hospital

On one hand Pakistan is facing severe energy crises, and on the other the planet is going through global warming. This has resulted in growing health issues, the only solution for both is low carbon planning. The purpose of this research is to show the effects of the windows of Civil burns hospital design. Moreover, to calculate the amount of UV light if it is sufficient for mood satisfaction. This research has taken into account analysis of ecological hospital designed with considerations of sun light. The mass of the building is built in a form to have the exact angle every hour for sufficient UV rays from the window. The efficiency with consideration to the mood satisfaction at Civil hospital. This has been examined in regard with window design and UV rays.

Literature suggests that adequate exposure to natural light provides a positive impact on human health and well being of patient and medical staff in a hospital environment. At Civil Hospital the pediatric headwall light is functioning as an ecological as well as healing for surgery daycare ward. In a daycare all activities are conducted all day long, hence little electricity lighting is used here, most of the natural full spectrum light is provided from the sky light. AKU is designed to give patients, visitors and staff contact with nature this may be a matter of physical access. Views of nature are known to be therapeutic. Gardens in healthcare environments are calming in themselves. Views from Skylight have mechanisms such as fostering access to social support and providing opportunities for positive escape and sense of control with respect to stressful clinical settings (Ulrich 2002). A peaceful nature scene is superlative in inducing feelings of calmness and safety (Ulrich 1984).

Firstly analysing the existing head wall light design of a surgical ward at civil on ECOTECT software. Secondly calculating the sun energy which is entering from the window. Thirdly, understanding the impact on mood satisfaction by comparing the answers from the questionnaire filled by patients. Finally, the shading devices of the eastern skylight at the Ambulatory ward are analysed on daily and annual basis on Ecotect.

It is a comparison of skylight on two typical days, of 
hospital's surgery ward. Firstly there is a complete analysis on $21^{\text {st }}$ June for an existing skylight that adopts traditional methods of achieving patient comfort through Mashrabiya. On the other hand a closed system with no windows and high energy consumption, in the same hospital design is considered.

The other objectives achieved here from the questionnaire were the following terms, and there explanation by the patients, staff and visitors.

1). Image, the visual concept of the building refers to and the ways of the building attracting attention to itself. The form of the building and the symbolic attributes

2). Community refers to how the building and its site represents a better hospital neighborhood and identity in terms of safety, security, and privacy.

3). Efficient functionality is an attribute in which the building is able to respond to the work which is held in process in it; and flow of people, equipment, and material.

4). Security here is defined as the level to which the building can segregate sensitive functions from one another and prevent the entry of people to restricted areas.

5). Flexibility is defined as the degree to which the building plan can be rearranged to conform to revised work progress and personnel changes.

7). Technical Performance refers to how the building operated in terms of mechanical systems, electrical systems and industrial processes.

8). Human performance is defined as how the building provides a physically and psychologically comfort to the residence, to work and live.

This paper shows another way of attaining comfort levels i.e. by constructing better windows and skylights for hospitals of Karachi. While the complex incorporates state-of-the-art educational and medical technologies, it also demonstrates that a modern architectural solution should preserve the spirit of its culture.

\subsection{Impact of sun light on human health and performance}

Finally there is a discussion on a model hospital window, considered as an Eco-Hospital window designed to take the advantage of green landscapes for obtaining the varying degree comfort levels as with the controlled methods. Research explores the role of design in improving the quality of patient environments to support the healing process and stimulate wellbeing. Questionnaire studies showed that bedridden patients assign especially high preference to having a hospital window view of nature [9-12]

Sun Light impacts human health and performance by two main mechanisms:

1. Controlling the body's circadian system

2. Affecting mood and alertness

In this section of paper, above two mechanisms is described and the specific impacts on human health and performance are outlined.
4.2.1. Analysis of the questions regarding the satisfaction regarding healthcare environments

The most important question arise here is "What do patients (and employees) need to see from the window" and 1) "Which architectural and atmospheric elements viewed from the window are perceived to enhance wellbeing and satisfaction"?

2). Secondly concerning the Healing Environments that should be more than 'just' designing a proper environment that reduces hospitalization duration or asking questions e. g. Can the views from Window reduce the hospitalization time.

3). Focusing on smooth care delivery processes (window and views production orientation)

4). Proposing a design for future: a more pleasant hospital window than present available.

\subsubsection{Analysis of the questionnaire from Nurse}

1). How do people classify window views in terms of their health status?

2). What is to be understood and treated about the windows?

3). The experience varies on the basic of a scientific diagnosis of the surgical disease of the patient vs. assessment of the overall state of health/illness of persons

4). How is suffering to be treated?

The search for the answer to the question "What explains movement toward the health: end of the health/illness?" led to the study of windows and views resources. Such resources are conceptualized in terms of the overall construct of the sense of orientation and meaningfulness.
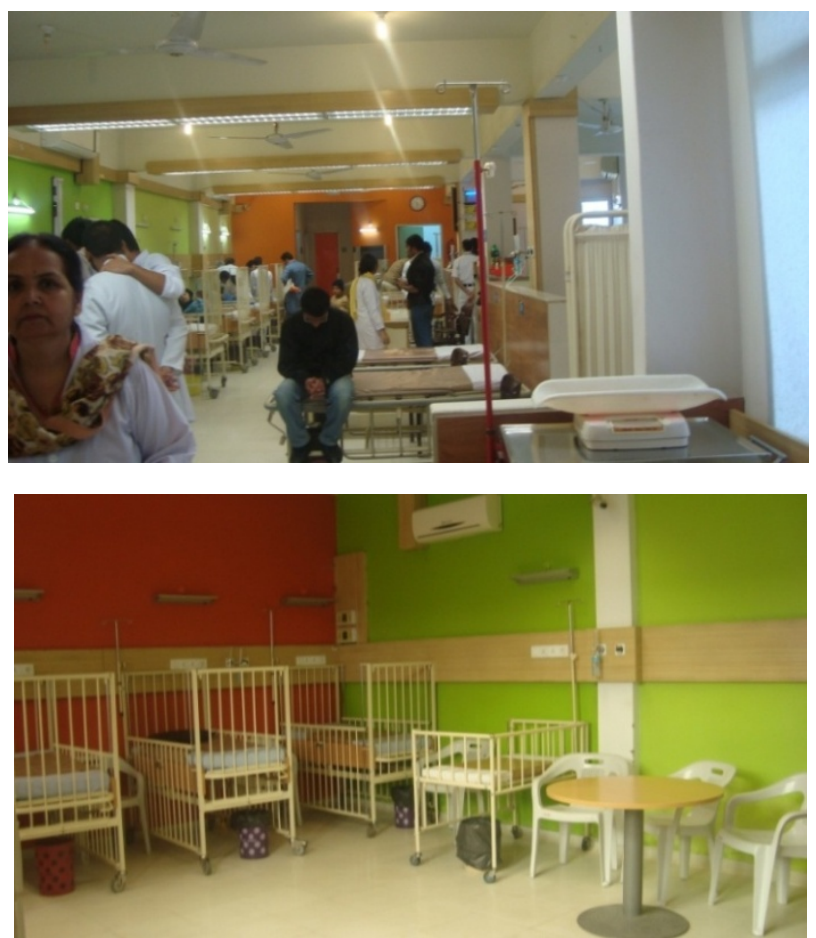

Figure 8. The interior of the mirror image wall as well as the same handed wall 


\subsection{Optimizing Natural Light without the Glare}

Patients can have enhanced privacy when they sleep on their side. Instead of facing the patient in the next bed, they can choose to face their own window. They can also draw the curtains, maintain good ventilation as well as enjoy a view of the outside through their windows

\subsubsection{Applied Analysis on Ambulatory ward: Thermal Analysis}

There are a number of approaches for zoning that can be applied to Hospital building when performing thermal analysis these are,

Firstly, a thermal zone represents an enclosed space within which the air is free to flow around and whose thermal conditions are relatively consistent. In most cases, any room that can be closed off with a door would be a separate zone.

Sometimes temperatures in different parts of large spaces can vary. In these cases, the space can be divided into a number of smaller zones with adjoining elements defined as voids. This way heat is free to flow between the zones, but their thermal characteristics can be analysed individually.

Also, adjacent utility spaces such as store rooms, toilets and corridors can often be grouped together into the one zone. This is because the exact temperature of each utility space is seldom of interest, but their action as a thermal buffer between other zones may be important.

In Figure 9 ECOTECT is used for shading and lighting calculations, the division of zones is not as important and they can be used as layers to separate objects and functions. However, thermal and acoustic calculations require very specific zoning based on the above rules. The building is assumed to be unoccupied and there is no air-conditioning system in operation at this time of analysis.

\subsection{Thermal Analysis on ECOTECT Model}

A model created for thermal analysis is geometrically simplified since the relevant attributes here are the thermo-physical properties; such as U-values and thermal admittance values. In the Computer Program ECOTECT if model of the building for the thermal calculation then it is also important to decide how to divide the building into appropriate zones, as shown in figure two a.

Since the objective here is to identify the mechanisms by which sun light impacts human health and performance and review the literature linking daylight with health outcomes in Pakistan healthcare settings. Hence in figure two The 3D Model structure is initially created in AutoCAD from measurements of the building. The user of the Ecotect has to be careful during the creation of layers for different elements of the building; such as walls, roofs, balconies, windows, and doors; for the layers creation might manipulate different features when exported to Ecotect, as shows in Figure12.

Nevertheless, the model exported from AutoCAD is manipulated in Ecotect so as to assign the different elements their respective characteristics and properties. The type of modelling used has to be compatible with the analysis performed. For example, solar shading and lighting analysis requires a model that contains all the necessary geometric information whereas a different type of model is needed for thermal simulations, as shown in (Figure 13).

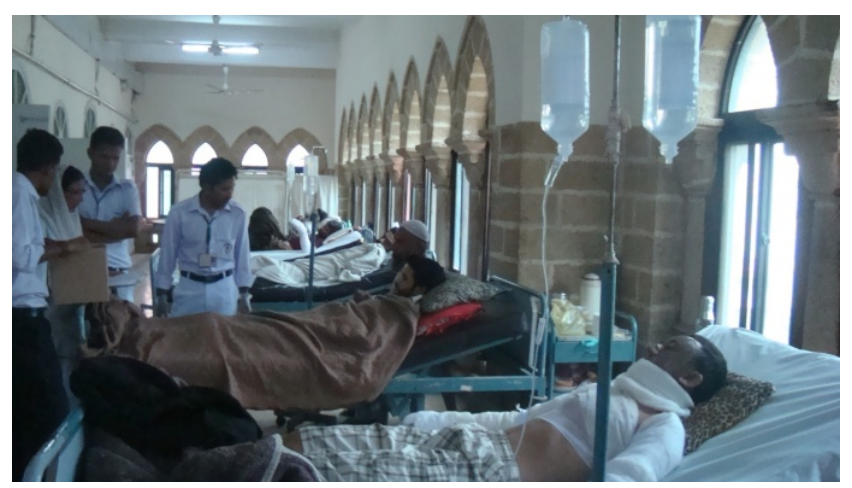

Figure 9. The burns center ward uses same handed as well as the mirror image head wall solution for the interior of the centralized nurse station nightingale ward

The warm summers in Karachi coupled with the intense high-altitude sunshine make passive solar control of skylight one of the important design considerations. The uncontrolled solar gain results in high cooling loads and excessive illumination, and also increases glare. The first strategy in sunlight and radiation passive cooling is solar heat gain avoidance, which can be achieved primarily through shading and selection of appropriate glazing devices e.g. Brise soleil.

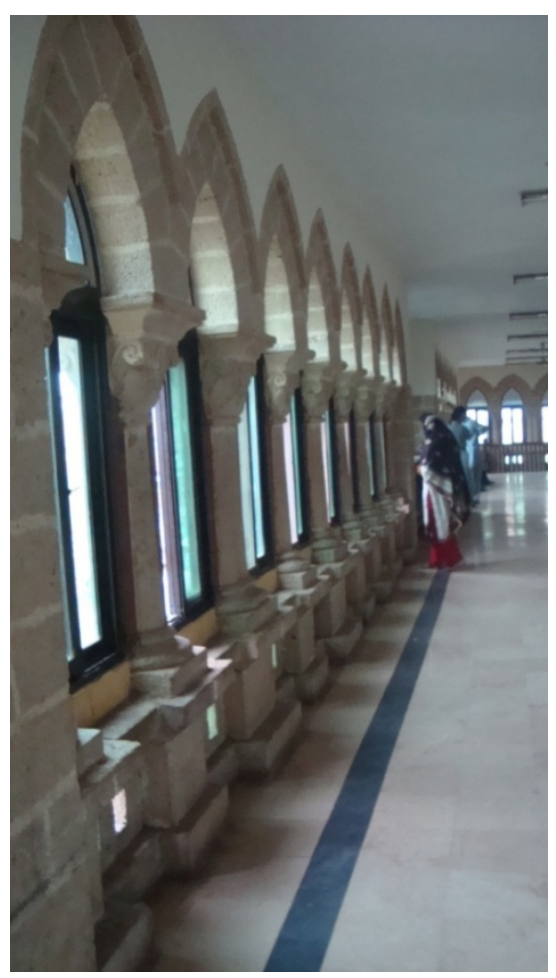

Figure10. the historic wall of burns center 
A recent randomized prospective study assessed whether the amount of sunlight in a hospital room modifies a patient's psycho-social health, quantity of analgesic medication used, and pain medication cost [14]. Patients undergoing elective cervical and lumbar spinal surgeries were admitted to the bright or the dim side of the same hospital unit postoperatively. The outcomes measured included the standard morphine equivalent of all opioid medication used postoperatively by patients and their subsequent pharmacy cost. Patients staying on the bright side of the hospital unit were exposed to $46 \%$ higher-intensity sunlight on average. This study found that patients exposed to an increased intensity of sunlight experienced less perceived stress, marginally less pain, took $22 \%$ less analgesic medication per hour, and had $21 \%$ less pain medication costs [14].

\section{Conclusion}

This paper analyzed the case studies of Karachi civil war came handed head wall and Civil hospital burns ward same handed centralized ward type based health facilities. The following determinants are applied on the case study analysis

\section{Conceptual framework}

The conceptual frame work derived from the literature review defined the scope of this paper on the basis of the following

Functionalism, technology then healing environment and evidence - based design plus sustainable design and now the multi disciplinary approach

The following conclusions were observed from the questionnaire.

Adequate and appropriate exposure to light is critical for health and well-being of patients as well as staff in healthcare settings.

Natural light should be incorporated into lighting design in healthcare settings, not only because it is beneficial to patients and staff, but also because it is light delivered at no cost and in a form that most people prefer.

Avoid building plans with large proportion of windowless rooms.

Suitable building orientation to provide access to natural lighting to improve patient and staff healthier outcomes.

\section{Acknowledgements}

We are very grateful to hospital experts for their appropriate and constructive suggestions to improve this research specially MS civil hospital Karachi.

\section{REFERENCES}

[1] Y. Stephen "The Future of the Patient Room:

[2] Challenges and Controversies" healthcare Magazine March 2009

[3] http://www.healthcaredesignmagazine.com/article/future-pat ient-room-challenges-and-controversies

[4] Practice Parameters Committee of the American College of Critical Care Medicine Society of Critical Care Medicine "Guidelines for Intensive Care Unit Design" 1995 Critical Care Medicine Mar; 23(3):582-588

[5] Sackett, DL; WMC Rosenberg; JA Muir Gray, RB Haynes \& WS Richardson "Evidence-Based Medicine: What it is and what it isn't" 1996 British Medical Journal 312: 71-2.

[6] The Quarterly published by Ellerbe Becket. "Health science edition" 2003Volume $4 \cdot$ number 4: 15 -16

[7] Rosalyn Cama, "Evidence-Based Healthcare Design" John Wiley \& Sons in association with the American Society of Interior Designers, 2009

[8] Dan R. Thompson; D. Kirk Hamilton; Charles D. Cadenhead; Sandra M. Swoboda; Stephanie M. Schwindel; Diana C. Anderson; Elizabeth V. Schmitz; Arthur C. St. Andre; Donald C. Axon; James W. Harrell; Maurene A. Harvey; April Howard; David C. Kaufman; Cheryl Petersen; "Guidelines for intensive care unit design" Critical Care Medicine 2012 Vol. 40, No. 5

[9] Ulrich, R. S, Zimring, C, Joseph, A, Quan, X, \&Choudhary, R. 2004. "The role of the physical environment in the hospital of the 21st century: A once-in-a-lifetime opportunity". Concord, CA: The Center for Health Design.

[10] Ulrich, R. S. 2002, "Health Benefits of Gardens in Hospitals", Center for Health Systems and Design

[11] Ulrich, R. S. 2000 "Effects of Healthcare Environmental Design on Medical

Outcomes"http://treebenefits.terrasummit.com/Documents/H ealth/Effects $\% 20$ of $\% 20$ Healthcare $\% 20$ environments.pdf

[12] Ulrich, R. S. 1986, "Effects of Interior Design on Wellness" - Major Hospital

Foundationhttp://www.majorhospitalfoundation.org/pdfs/Eff ects\%20of\%20Interior\%20Design \%20on\%20Wellness.pdf

[13] Ulrich, R. S. 1984 "View through a window may influence recovery from surgery". American Association for the Advancement of Science

[14] Walch, J. M., Rabin, B. S., Day, R., Williams, J. N., Choi, K., \& Kang, J. D. 2005 "The effect of sunlight on post operative analgesic medication usage: A prospective study of spinal surgery patients. Psychosomatic Medicine,67(1), 156-163 